\title{
KidNet: An Automated Framework for Renal Lesions Detection and Segmentation in CT Images *
}

\author{
Sulaiman $\left.\operatorname{Vesal}^{1}(凹)\right)^{[0000-0001-6156-9338]}$, Nishant Ravikumar ${ }^{1}$, and Andreas K. \\ Maier $^{1}$ \\ 1 Pattern Recognition Lab, Friedrich-Alexander-Universität Erlangen-Nürnberg, \\ Germany \\ 2 CISTIB, Centre for Computational Imaging and Simulation Technologies in \\ Biomedicine, School of Computing, University of Leeds, LS2 9JT, United Kingdom \\ sulaiman.vesal@fau.de
}

\begin{abstract}
Renal lesions segmentation and morphological assessment are essential for improving diagnosis and our understanding of renal cancer, which in turn is imperative for reducing the risk of mortality and morbidity in patients. In this paper, we propose an automatic imagebased method to first detect kidneys in CT images and then segment both kidneys and lesions in higher resolution. Kidneys are detected using an encoder-decoder method trained on low-resolution images. Based on probability maps generated by detector model, we can identify corresponding kidney regions and segment both kidneys and lesions in higherresolution with reducing the false positive voxels. We evaluate our approach on KITS 2019 challenge data set and demonstrate that our proposed method generalizes to unseen clinical CTs of the abdominal.
\end{abstract}

Keywords: First keyword - Second keyword - Another keyword.

\section{Introduction}

\section{Experiments and Results}

\section{Discussion and Conclusions}

\section{Acknowledgments.}

\section{References}

* Supported by Erlangen EFI project. 